\title{
Molecular third-harmonic-generation microscopy through resonance enhancement with absorbing dye
}

\author{
Che-Hang Yu, ${ }^{1}$ Shih-Peng Tai, ${ }^{1}$ Chun-Ta Kung, ${ }^{1}$ Wen-Jeng Lee, ${ }^{1,2}$ Yi-Fan Chan, ${ }^{3}$ Hsiang-Lin Liu, ${ }^{4}$ \\ Jiun-Yi Lyu, ${ }^{4}$ and Chi-Kuang Sun ${ }^{1,5, *}$ \\ ${ }^{1}$ Department of Electrical Engineering and Graduate Institute of Photonics and Optoelectronics, \\ National Taiwan University, Taipei 10617, Taiwan \\ ${ }^{2}$ Department of Medical Imaging, National Taiwan University Hospital, Taipei 10617, Taiwan \\ ${ }^{3}$ Tissue Bank Core Laboratory, National Taiwan University Center for Genomic Medicine, Taipei 10617, Taiwan \\ ${ }^{4}$ Department of Physics, National Taiwan Normal University, Taipei 11677, Taiwan \\ ${ }^{5}$ Research Center for Applied Sciences, Academia Sinica, Taipei 115, Taiwan \\ *Corresponding author: sun@cc.ee.ntu.edu.tw
}

Received September 18, 2007; revised November 21, 2007; accepted January 7, 2008; posted January 14, 2008 (Doc. ID 87608); published February 14, 2008

\begin{abstract}
This paper reports a facile and effortless method to realize three-dimensional (3D) molecular thirdharmonic-generation (THG) microscopy through the technique of resonance enhancement with absorbing dye. Hematoxylin, a popular absorbing stain, is applied as an example to verify the multiphoton resonant enhancement based on the $1230 \mathrm{~nm}$ excitation light and can selectively enhance THG yield at cell nuclear sites in the studied specimens, serving as a cell nucleus contrast agent. It is concluded that combining THG microscopy with the mature staining technique can readily achieve 3D molecular imaging without using fluorescence. (C) 2008 Optical Society of America

OCIS codes: $170.6900,190.4160,190.4180$.
\end{abstract}

In recent years, the contrast mechanisms of the virtual-transition-based third-harmonic-generation (THG) process have been progressively understood, and subcellular imaging originating from intrinsic THG sources has been demonstrated in unstained cells and tissues. With the advantages of minimal invasiveness and high three-dimensional (3D) resolution, THG microscopy, based on endogenous contrast, has been applied for morphological visualizations of neurons [1], muscle fibers [2], elastic fibers [3], lipid bodies [4], red blood cells [5], embryos [6], skins [7], and the oral cavity [8]. What seems to be lacking is the capability for THG as a molecular imaging tool. The motivation to develop exogenous THG contrast agents to trace the function of a specific molecule is high because of their advantages of high 3D resolution and no involvement of electron transition, and therefore no energy deposition and thus no induced photobleaching and photodamage, when compared with regular fluorescence or absorption contrast based microscopy. By using metal nanoparticles as an exogenous contrast agent, plasmonresonance-enhanced THG microscopy was recently demonstrated with a molecular specificity [9]. This success has driven increasing interest in finding other external THG contrasts that can provide molecular and functional information on biotissues.

In this paper, we present a facile and effortless method for 3D molecular THG imaging through resonance enhancement with absorbing dye. Resonance enhancement of THG means that the magnitude of the nonlinear THG susceptibility can be greatly enhanced if one of the electron transition frequencies of a specific molecule closely matches one of the virtual transition frequencies of the THG process, including single-photon, two-photon, and three-photon frequencies $[10,11]$. Staining is a well-developed technique that is regularly performed in biology and medicine to highlight or classify cells or structures with contrasting colors by means of absorbing stains. Absorbing stains are minute colored substances with electron transitions that absorb some preferential wavelengths of light and have affinities to some specific molecules, and they are widely used for conventional microscopes not only in vitro but also in vivo. Compared with the complex surface process of metal nanoparticles, the use of absorbing stains has the advantages of ready availability and convenience. By choosing absorbing wavelengths of molecular stains that are nearly coincident with one of the virtual levels of the THG process, molecular THG imaging should be easily achievable through resonance enhancement. Taking the most popular stain, hematoxylin stain, as an example, here we demonstrate 3D biomolecular THG microscopy by using staining. The molecular specificity of the THG microscopy can be simply identified through the comparison between the images before and after the staining enhancement.

Hematoxylin stain, which is the most widely used stain and takes only a few minutes for staining, stains mainly basophilic structures containing nucleic acids with a blue-purple hue, such as the chromatin-rich cell nuclei for medical diagnosis. Figure 1(a) shows the measured absorption spectrum of hematoxylin. It has a broad range of absorption at the visible light band, achieving the two- and threephoton resonance enhancement criteria [10] of the THG process with a $1230 \mathrm{~nm}$ incident wavelength [illustrated in Fig. 1(b)], while avoiding the excitation 


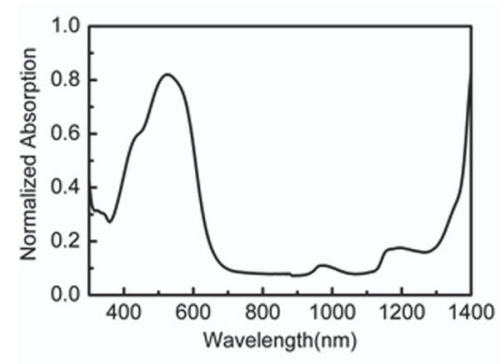

(a)

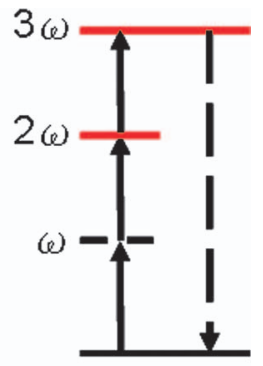

(b)
Fig. 1. (a) Absorption spectrum of hematoxylin dissolved in water. (b) Schematic diagram showing the two- and three-photon resonant enhancement of the THG process.

light absorption. Therefore, one would expect to see selectively enhanced THG signals after the usage of hematoxylin. This study was performed with a homebuilt epi-THG microscope [8] with a backward collection geometry to assist future clinical examinations. The detailed optical geometry can be found in [8]. The excitation laser was a Cr:forsterite laser centered at $1230 \mathrm{~nm}$ with a $110 \mathrm{MHz}$ repetition rate and a $100 \mathrm{fs}$ pulse width. The average laser power on the surface of the studied specimens was kept constant at $150 \mathrm{~mW}$. We cut the fresh human lung tissues into $25 \mu \mathrm{m}$ thick sections. The tested specimens were first imaged by conventional microscopy, followed by the epi-THG microscopy, then stained by hemotoxylin for about $1 \mathrm{~min}$, imaged by the epi-THG microscopy, and finally imaged by conventional microscopy again. This protocol was approved by the Research Ethics Committee of the National Taiwan University Hospital. Figure 2 is the multimodal epi-second-harmonicgeneration (epi-SHG) and epi-THG images of the normal human lung tissues before and after hematoxylin staining, along with the images of conventional microscopy for comparison. To compare with the conventional microscopy images, the THG images shown in Fig. 2 were stacked by 25 depthdependent horizontal epi-THG sections with $1 \mu \mathrm{m}$ depth difference between, corresponding to a $\sim 25 \mu \mathrm{m}$ effective thickness. Before staining [Fig. 2(a)], THG contrast mainly originates from endogenous elastic fibers and lipid bodies [3,4], while the THG signals from the cell nucleus membrane are much weaker. After they were stained with hematoxylin, some ovoid structures showed up in the THG images. Compared with conventional microscopy, these locations can be confirmed to be cell nuclei, indicating that hematoxylin resonantly enhanced the THG signals. The THG signals from the cell nuclei were resonantly enhanced about 2 orders of magnitude and can be at least two times higher than the strong endogenous THG signals from the elastic fibers, while Fig. 2(b) has to be taken at low PMT voltage to avoid PMT detection saturation. Figure 2(c) shows the overlapped image of Figs. 2(a) and 2(b), implying the origins of THG contrasts can be exogenously manipulated to offer different histological information. Through this merged image, false positive signals due to endogenous background can be identified. Figure 3 shows the depth-resolved horizontal

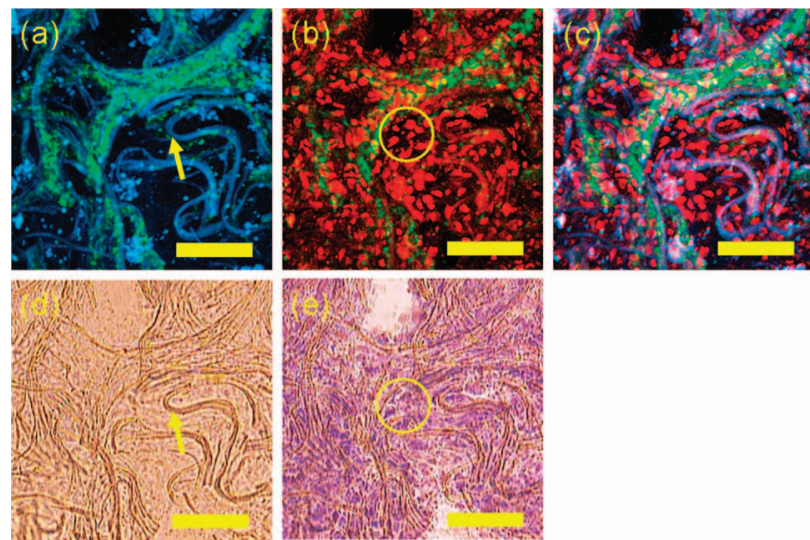

Fig. 2. (a) Combined epi-THG (blue)/epi-SHG (green) image of the nomal human lung before staining. THG signals from curvilinear elastic fibers (arrow) and dotlike lipid bodies are identified. SHG signals come from collage fibers in alveolar walls. Photomultiplier voltage for THG: $1200 \mathrm{~V}$. (b) Combined epi-THG (red)/epi-SHG (green) image after 1 min hematoxylin staining. Multiple ovoid-shaped cell nuclei (circle) are observed. Photomultiplier voltage for THG: 1000 V. (c) Overlaped image of (a) and (b). (d) and (e) Corresponding conventional images of (a) and (b). The color of the cell nuclei after hematoxylin stain is blue-purple in conventional microscopy. Scale bar: $50 \mu \mathrm{m}$.

sections of the epi-SHG and epi-THG images in normal human lung tissue, indicating the capability of direct acquisition of images with excellent axial resolution, which is not offered by conventional absorption microscopy. Owing to virtual transitions, the resonance-enhanced THG process itself is without the problems of bleaching and absorption. With the generated THG intensity lower than the nanowatt level, stain absorption and bleaching by the generated THG are also negligible. According to our experimental results, no THG photodamage or photobleaching phenomena could be observed in the stained samples even under $150 \mathrm{~mW}$ average power illumination during the whole experimental process.

With a strongly enhanced THG contrast from cell nuclei through hematoxylin staining, one should be able to see the different spatial distributions of the cell nuclei between normal and cancerous tissues, while the cell nuclear morphology is a key issue for cancer diagnosis. Taking advantage of the cell nuclear visualization capability, we characterized normal and cancerous human lung tissues through hemotaxylin-stained epi-THG microscopy. Surgical
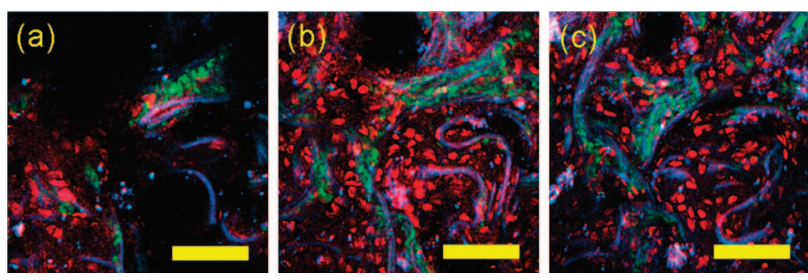

Fig. 3. Depth-resolved epi-SHG/epi-THG images of the normal human lung tissue at depths of (a) $8 \mu \mathrm{m}$, (b) $16 \mu \mathrm{m}$, and (c) $24 \mu \mathrm{m}$. Epi-SHG/epi-THG signals before hematoxylin staining are denoted by green/blue. Epi-THG signals after hematoxylin staining are denoted by red. Scale bar: $50 \mu \mathrm{m}$. 

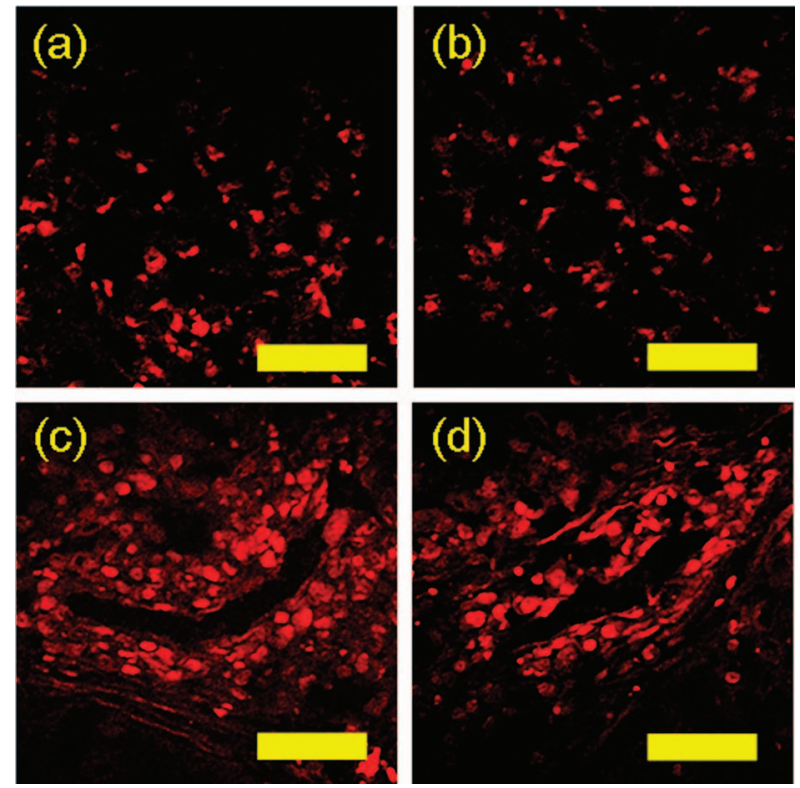

Fig. 4. Hematoxylin-stained epi-THG images of two surgical resected human lung cancer (adenocarcinoma) specimens. (a) and (c) Normal and cancerous parts of the first specimen, respectively. (b) and (d) Normal and cancerous parts of the second specimen, respectively. The size of the cell nuclei is enlarged in the cancerous part, and the cancerous cells are arranged in a circular pattern (acinar pattern), typical for adenocarcinoma. Scale bar: $50 \mu \mathrm{m}$.

resected lung specimens from two patients with lung cancer were evaluated in this study. We examined normal and cancerous parts of the resected human lung tissues stained by hemotaxylin in epi-THG microscopy and conventional microscopy. Figure 4 shows the comparison of the hematoxylin-enhanced epi-THG images of normal and cancerous lung tissues. In Fig. 4, we can distinguish the cancerous from the normal human lung tissues according to the typical morphology for adenocarcinoma with enlarged cell nuclei size and a circular pattern (acinar pattern) in the neoplastic tissue. With the capability to penetrate into the resected specimen for 3D nucleus imaging without the time-consuming fixing, paraffin embedding, and microtome cutting in routine histopathology procedures, this developed technique could provide rapid preliminary evaluation to determine the sectioned margin during operation, while the hematoxylin stain is compatible with the subsequent pathology procedure.
In summary, we demonstrate molecular THG microscopy utilizing nonfluorescent absorbing dye. By using stain-absorbing levels that coincide with the multiphoton frequencies of the excitation light, we demonstrate that hematoxylin stain can selectively enhance THG yield at cell nuclei in the studied specimens, serving as a cell nucleus contrast agent. Selectively enhanced THG signals provide immediate 3D cell nuclei images owing to the intrinsic optical sectioning capability. Without tedious fixation and sectioning procedures, epi-THG imaging with stain is simple to perform, takes only a few minutes, and can reduce artificial damage to valuable clinical specimens. If one carefully chooses the absorption property to match the criterion of the resonance enhancement and with the specific molecular affinity, this technique should be widely applicable to 3D biomolecular imaging without using fluorescence.

This work is sponsored by the National Health Research Institute (NHRI-EX96-9201EI) of Taiwan, an NTU Frontier Research Grant, and the NTU Center for Medical Excellence.

\section{References}

1. D. Yelin and Y. Silberberg, Opt. Express 5, 169 (1999).

2. S.-W. Chu, S.-Y. Chen, G.-W. Chern, T.-H. Tsai, Y.-C. Chen, B.-L. Lin, and C.-K. Sun, Biophys. J. 86, 3914 (2004).

3. C.-H. Yu, S.-P. Tai, C.-T. Kung, I.-J. Wang, H.-C. Yu, H.-J. Huang, W.-J. Lee, Y.-F. Chan, and C.-K. Sun, Opt. Express 15, 11167 (2007).

4. D. Debarre, W. Supatto, A.-M. Pena, A. Fabre, T. Tordjmann, L. Combettes, M.-C. Schanne-Klein, and E. Beaurepaire, Nat. Methods 3, 47 (2006).

5. R. D. Schaller, J. C. Johnson, and R. J. Saykally, Anal. Chem. 72, 5361 (2000).

6. C.-K. Sun, S.-W. Chu, S.-Y. Chen, T.-H. Tsai, T.-M. Liu, C.-Y. Lin, and H.-J. Tsai, J. Struct. Biol. 147, 19 (2004).

7. C.-K. Sun, C.-C. Chen, S.-W. Chu, T.-H. Tsai, Y.-C. Chen, and B.-L. Lin, Opt. Lett. 28, 2488 (2003).

8. S.-P. Tai, W.-J. Lee, D.-B. Shieh, P.-C. Wu, H.-Y. Huang, C.-H. Yu, and C.-K. Sun, Opt. Express 14, 6178 (2006).

9. S.-P. Tai, Y. Wu, D.-B. Shieh, L.-J. Chen, K.-J. Lin, C.-H. Yu, S.-W. Chu, C.-H. Chang, X.-Y. Shi, Y.-C. Wen, K.-H. Lin, T.-M. Liu, and C.-K. Sun, Adv. Mater. (Weinheim, Ger.) 19, 4520 (2007).

10. R. B. Boyd, Nonlinear Optics, 2nd ed. (Academic, 2003).

11. G. Veres, S. Matsumoto, Y. Nabekawa, and K. Midorikawa, Appl. Phys. Lett. 81, 3714 (2002). 